\title{
Analysis on full-domain apparent resistivity characteristics of ground-tunnel transient electromagnetic method
}

\author{
Yaoning Liu ${ }^{1}$, Shucai Liu ${ }^{2, *}$, Zhihai Jiang ${ }^{2}$, and Shuangshuang Chen ${ }^{2}$ \\ ${ }^{I}$ Institute of Building Intelligence, Jiangsu Vocational Institute of Architectural Technology, Xuzhou, China \\ ${ }^{2}$ The School of Resource and Geoscience, China University of Mine and Technology, Xuzhou, China
}

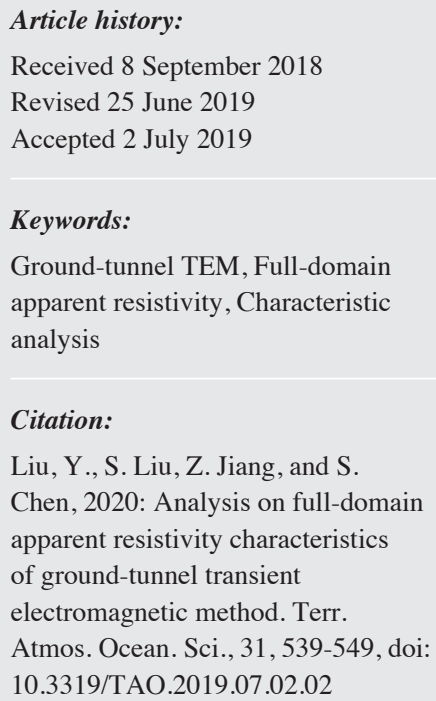

Liu, Y., S. Liu, Z. Jiang, and S. Chen, 2020: Analysis on full-domain apparent resistivity characteristics of ground-tunnel transient electromagnetic method. Terr. Atmos. Ocean. Sci., 31, 539-549, doi: 10.3319/TAO.2019.07.02.02

\begin{abstract}
The ground-tunnel transient electromagnetic method (TEM) is a geophysical prospecting method in which electromagnetic waves are emitted into the ground and measurements are taken in an underground tunnel. Based on the monotonic relationship between vertical magnetic induction intensity and apparent resistivity, this paper puts forward an idea for finding a unique solution and/or multiple solutions of the full-domain apparent resistivity of ground-tunnel TEM according to the inverse function theorem. It mainly involves calculating and analyzing the response characteristics of the full-domain apparent resistivity curves of four different geoelectric models $\mathrm{H}, \mathrm{K}, \mathrm{A}$, and $\mathrm{Q}$ in different positions to changes in such parameters as formation resistivity. The results show that full-domain apparent resistivity calculated using vertical magnetic induction intensity can accurately reflect the electrical distribution laws of different geoelectric models. When the formation parameters and receiving point positions change, the full-domain apparent resistivity curve shape changes accordingly. The change is specifically expressed in the time order and amplitude, and can accurately reflect formation information. Finally, combined with the characteristics of ground-tunnel TEM, full-domain apparent resistivity with different offsets is calculated. The results show that full-domain apparent resistivity curves with different offsets are consistent, with little differentiation. The analysis of the full-domain apparent resistivity of ground-tunnel TEM can lay a theoretical foundation for the interpretation of this method.
\end{abstract}

\section{INTRODUCTION}

With the increase in coal mining depth, the hydrogeological conditions of mines are becoming more and more complicated, and coal mine water inrush disasters seriously threaten the safe production of coal mines (Wu 2014). As a low-frequency electromagnetic geophysical prospecting method, the transient electromagnetic method (TEM) is widely used for hydrogeological disaster prediction in coal mines (Xue et al. 2012, 2018a, b, c; Jiao et al. 2016). In ground TEM, as it is located far from the hydrogeological target, resolution cannot be assured in either the horizontal and vertical direction. Mine TEM is widely used in the prediction of goaf water, column collapse and mine disaster water sources, but some problems remain such as the coil

\footnotetext{
* Corresponding author

E-mail:liushucai@cumt.edu.cn
}

mutual inductance problem, full space effect and metal interference in the tunnel (Li et al. 2013; Liu 2014; Zhou et al. 2014; Chang et al. 2016). Ground-tunnel TEM (Meng and Pan 2012; Li et al. 2015, 2016; Jiao 2016) is proposed by the integrating the advantages of both ground TEM and mine TEM. This method achieves high power transmission while realizing such advantages as the closer distance of the receiving device to the target, good coupling, large exploration depth and high resolution of abnormal bodies. However, due to the separation of the field source and observation system in the vertical direction, the spatial distribution characteristics of the observed secondary field are different from those of ground and mine TEM. The interpretation of the reconnaissance data is also different from that of data obtained using ground and mine TEM.

Apparent resistivity is the most widely used interpretative parameter in TEM (Wang et al. 2019; Yen et al. 2019). 
In the data analysis, the later-stage apparent resistivity of the central loop is commonly used for calculation. However, when the approximate conditions are not satisfied, the apparent resistivity curve will be distorted and contain errors. As the offset increases, the error gradually increases (Qiang et al. 2010; Qi et al. 2011a, b). Full-domain apparent resistivity can effectively solve this problem. Bai Chenghai and Zhu Ningjun proposed a numerical calculation method for calculating the whole resistivity of the central loop using the kernel function (Bai et al. 2003; Zhu 2015). Cui Jiangwei calculated full-domain apparent resistivity based on the relationship between magnetic induction intensity and resistivity (Cui et al. 2015). These studies have played an important promotional role in the accurate interpretation of transient electromagnetic data.

Both the measurement points and emission loops of ground-tunnel TEM have offsets in both the vertical and horizontal direction. At present, there is no published analysis of the apparent resistivity curve characteristics of the method, so it is necessary to study the resistivity characteristics of ground-tunnel TEM in different geoelectric models in order to gain a more intuitive and reliable interpretation method for the reconnaissance method.

\section{FULL-DOMAIN APPARENT RESISTIVITY CALCULATION OFGROUND-TUNNEL TEM}

\subsection{Analysis of Vertical Magnetic Induction Intensity and Induced Electromotive Force Response Characteristics}

Apparent resistivity is usually defined in two way: by vertical magnetic induction intensity $\left(B_{z}\right)$ and by the time derivative of vertical magnetic induction intensity $\left(\partial B_{z} / d t\right)$ (induction electromotive force). With the development and promotion of fluxgate probes, it is possible to directly calculate apparent resistivity using vertical magnetic induction intensity. Many scholars have also verified the advantages of defining apparent resistivity using magnetic field intensity (Chen et al. 2012; Zhao et al. 2016). The vertical magnetic field of the rectangular return line can be obtained via the numerical integration of the TE expression of the horizontal electric dipole along the return line (Li et al. 2011). The frequency domain and time domain conversion can be obtained from the sine and cosine variations.

Figure 1 is a schematic of a rectangular loop with a length and width of $2 \mathrm{~L}$ and $2 \mathrm{~W}$ respectively. The coordinate origin $(0,0,0)$ is located at the geometric centre of the rectangular loop, and the $\mathrm{Z}$ axis is vertically downward. The small horizontal galvanic source coordinates are $\left(x^{\prime}, y^{\prime}, 0\right)$ and the receiving point coordinates are $(x, y, z)$, flowing in a clockwise direction, the current I is passed through the loop.

The vertical magnetic induction at the $(x, y, z)$ point can be obtained by superimposing the magnetic fields excited by the $\mathrm{AB}$ side, $\mathrm{BC}$ side, $\mathrm{CD}$ side, and $\mathrm{DA}$ side.

$$
\begin{aligned}
& B_{z}= \\
& -\frac{I \mu_{0}}{4 \pi} \int_{-L}^{L} \int_{0}^{\infty} \frac{\lambda^{2}}{u_{0}}\left[a_{n} e^{-u_{n}\left(z-z_{n}\right)}+b_{n} e^{u_{n}\left(z-z_{n+1}\right)}\right] \\
& \quad\left\{\frac{W-x}{\rho_{W}} J_{1}\left(\lambda \rho_{W}\right)-\frac{-W-x}{\rho_{W}} J_{1}\left(\lambda \rho_{-W}\right)\right\} d \lambda d y^{\prime} \\
& -\frac{I \mu_{0}}{4 \pi} \int_{-W}^{W} \int_{0}^{\infty} \frac{\lambda^{2}}{u_{0}}\left[a_{n} e^{-u_{n}\left(z-z_{n}\right)}+b_{n} e^{u_{n}\left(z-z_{n+1}\right)}\right] \\
& \quad\left\{\frac{L-y}{\rho_{L}} J_{1}\left(\lambda \rho_{L}\right)-\frac{-L-y}{\rho_{-L}} J_{1}\left(\lambda \rho_{-L}\right)\right\} d \lambda d x^{\prime}
\end{aligned}
$$

Where $I$ is the current intensity, $\rho_{-L}=\left[\left(x^{\prime}-x\right)^{2}+(-L-y)^{2}\right]^{1 / 2}$, $\rho_{W}=\left[(W-x)^{2}+\left(y^{\prime}-y\right)^{2}\right]^{1 / 2}, \quad \rho_{L}=\left[\left(x^{\prime}-x\right)^{2}+(L-y)^{2}\right]^{1 / 2}$,

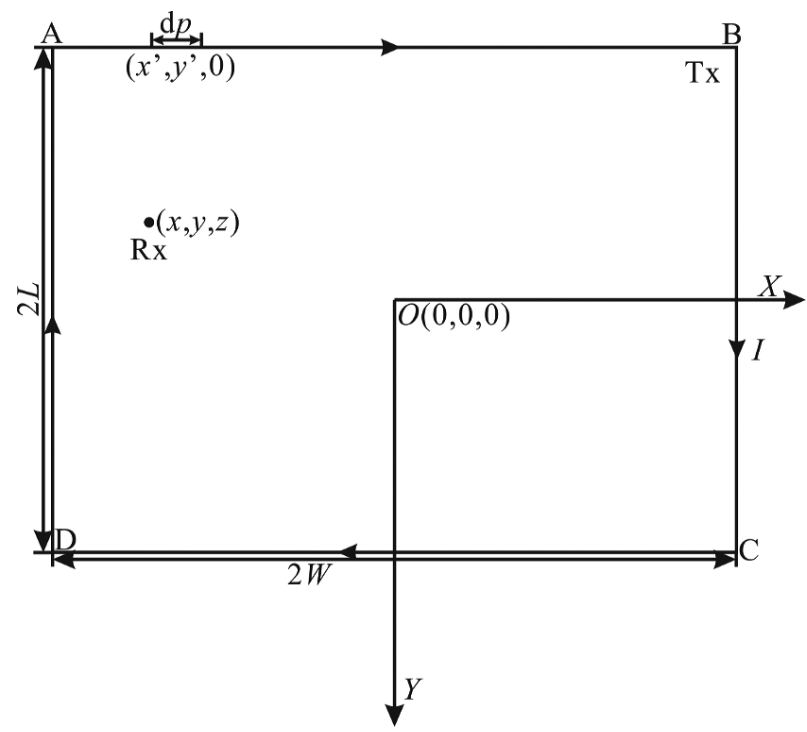

Fig. 1. Schematic of rectangular loop. 
$\rho_{-W}=\left[(-W-x)^{2}+\left(y^{\prime}-y\right)^{2}\right]^{1 / 2}, W$ and $L$ are the half-length and half-width of the rectangular loop, $\lambda$ is the wave number, $J_{0}$ is the zero-order Bessel function, $J_{1}$ is the firstorder Bessel fuction, $a_{n}$ and $b_{n}$ are amplitude coefficients.

Figure 2 is a schematic diagram of a uniform geodetic model and a ground-tunnel TEM observation system. Model parameters: the rectangular emission loop is $500 \mathrm{~m}$ in length and $500 \mathrm{~m}$ in width, and the emission current is $1 \mathrm{~A}$. The current direction is shown by the arrow in the figure. The origin of the coordinate system is at the centre of the rectangular loop. The coordinate system is as shown in the figure. The observation coordinates are $\operatorname{Rx}(x, y, z)$ with unit in meters.

Figure 3 shows the variation curve of the receiving point responses of the ground-tunnel TEM at different depth $\partial B_{z} / d t$ responses and $B_{z}$ responses at different times changing with the change of the uniform geoelectric model resistivity. It can be seen from the figure that, the curve of $\partial B_{z} / d t$ changing with the resistivity is not a monotonic, and the curve shape is comparatively complicated. With the increase of the receiving point depth, no effective $\partial B_{z} / d t$ value can be obtained at the early stage; and $B_{z}$ is a monotonic function when changing with the resistivity, but the resolution of the early stage data is low.

Figure 4 shows the variation curve of $\partial B_{z} / d t$ and $B_{z}$ 's response at different moments to the uniform geodetic model resistivity change when the ground-tunnel TEM receiving points are at the same depth but with different offsets. With the change of the offset, the $\partial B_{z} / d t$ curve shape shows no obvious change, and the $\partial B_{z} / d t$ response is still a non-monotonic function with the resistivity; although the $B_{z}$ curve shape is simple, as the offset changes, the curve shape first rises and then falls; and when there is an offset, the response is also a non-singular value function of resistivity.

\subsection{Calculation of Full-Domain Apparent Resistivity}

According to Yin and Piao (1991) and inverse function theory, this paper considers the relationship between vertical magnetic induction $B_{z}$ and the resistivity parameter as a two-valued function consisting of two monotonic functions.
Figure 5 shows the apparent resistivity calculation process of Ground-Tunnel TEM. For a function with a unique solution, if the $B_{z}$ response is the monotonic function of the resistivity, it can be known that a unique resistivity corresponds to the $B_{z}$ value according to the inverse function theorem. The Taylor formula is used to expand the $B_{z}$ integral expression into series form to obtain the main linear part, establish the iteration relation of apparent resistivity and arrive at the solution in this paper.

For a function with two solutions, the dichotomy method (Cui et al. 2015; Zhao et al. 2016) is used to calculate the extreme point $\rho_{\text {sextre }}$ within the given resistivity range, calculate $\rho_{s 1}$ and $\rho_{s 2}$ respectively within the resistivity value ranges $\left(\rho_{\text {smin }}, \rho_{\text {sextre }}\right)$ and $\left(\rho_{\text {sextre }}, \rho_{\text {smax }}\right)$ and select a proper value as the final solution $\rho_{s}$.

To validate the effectiveness of the above calculation concept of the full-domain apparent resistivity of groundtunnel TEM, the uniform earth model is calculated as a trial. The model's parameters are described as follows: uniform earth resistivity is $\rho=100 \Omega \cdot \mathrm{m}$, the transmission current is $1 \mathrm{~A}$, the rectangular loop is $500 \mathrm{~m}$ in both length and width, the time window is $10^{-5}-10^{-1} \mathrm{~s}$, the coordinate system origin is located at the center of the rectangular loop and the measurement point coordinate is marked as $\mathrm{Rx}$.

Figure 6 shows that the ground-tunnel full-domain apparent resistivity used to calculate the vertical magnetic induction intensity approximates to the true uniform earth resistivity and the error of the relative error curve pair is less than 5\%, which indicates that the calculation method of the full-domain apparent resistivity of ground-tunnel TEM defined using vertical magnetic induction intensity is feasible.

\section{RESULTS ANALYSIS OF FULL-DOMAIN APPARENT RESISTIVITY CURVE CHARACTERISTICS OF GROUND-TUNNEL TEM}

To further analyse the full-domain apparent resistivity curve characteristics of ground-tunnel TEM and provide foundational information in the actual exploration process,

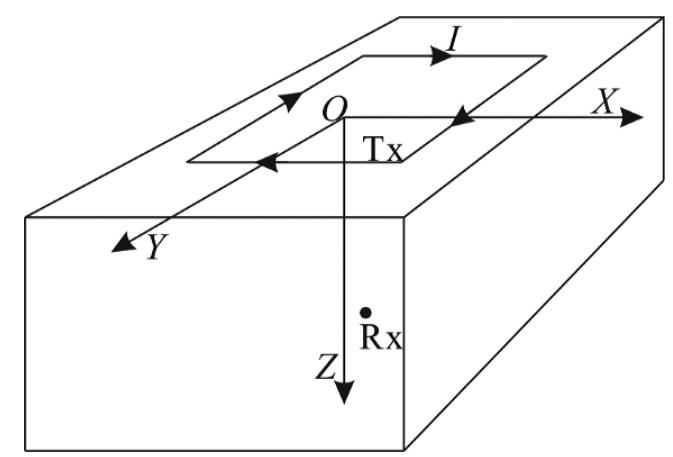

Fig. 2. The uniform geodetic model and Ground-Tunnel TEM observation system. 

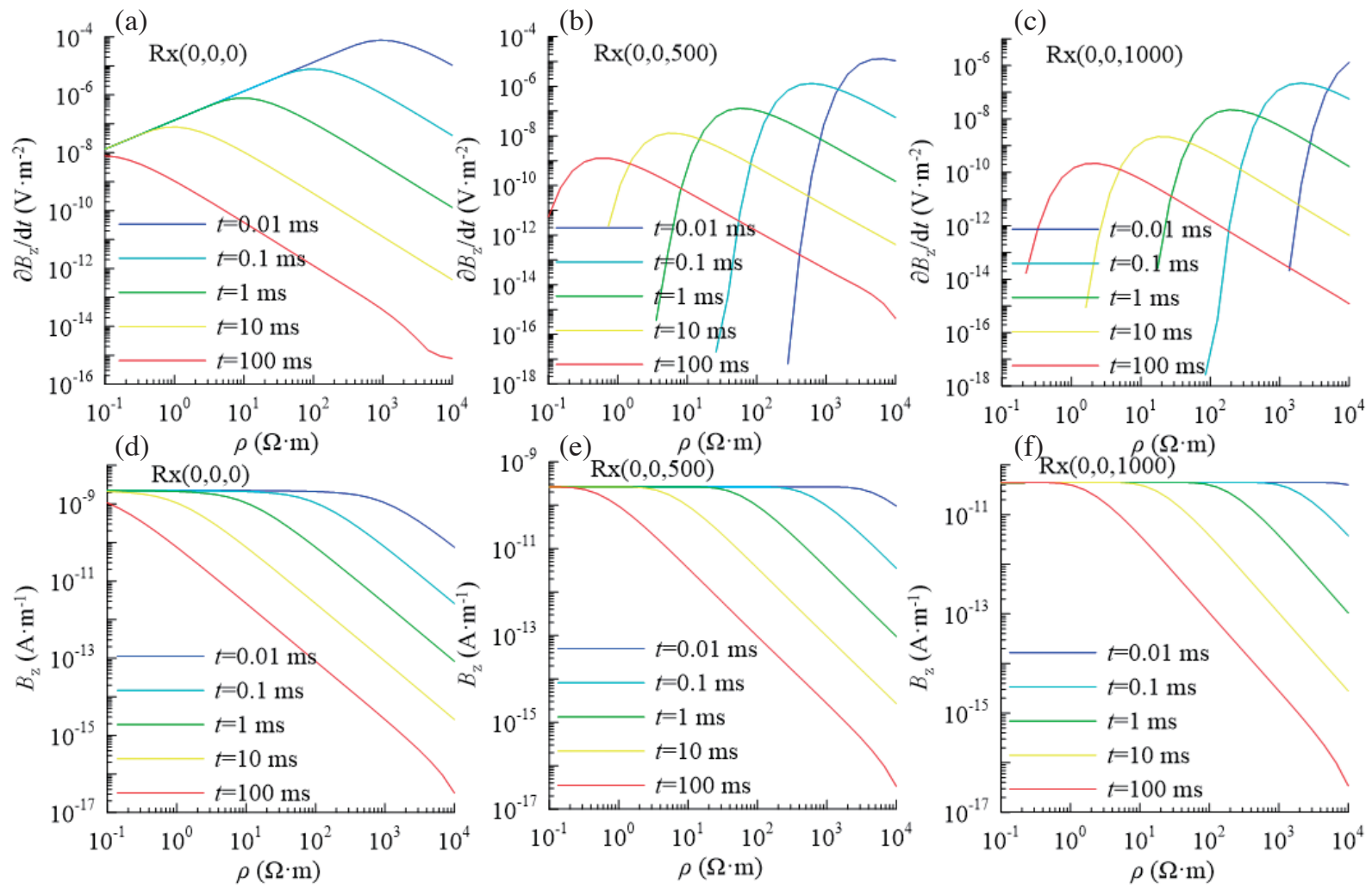

Fig. 3. Response curves of $\partial B_{z} / d t$ and $B_{z}$ receiving point at different depth; (a) $\operatorname{Rx}(0,0,0), \partial B_{z} / d t ;(\mathrm{b}) \operatorname{Rx}(0,0,500), \partial B_{z} / d t ;(\mathrm{c}) \operatorname{Rx}(0,0,1000)$, $\partial B_{z} / d t ;$ (d) $\operatorname{Rx}(0,0,0), B_{z} ;$ (e) $\operatorname{Rx}(0,0,500), B_{z} ;$ (f) $\operatorname{Rx}(0,0,1000), B_{z}$.

(a)
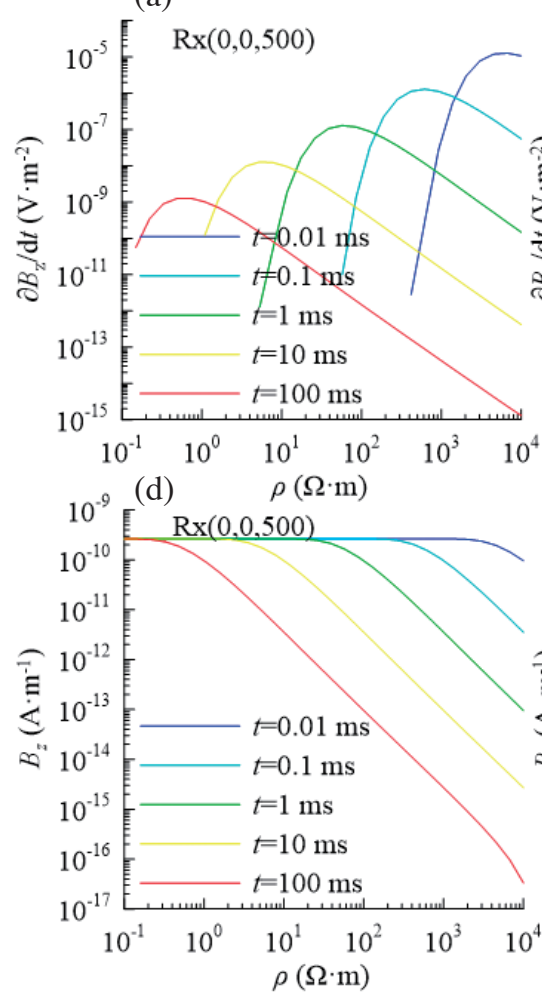

(b)

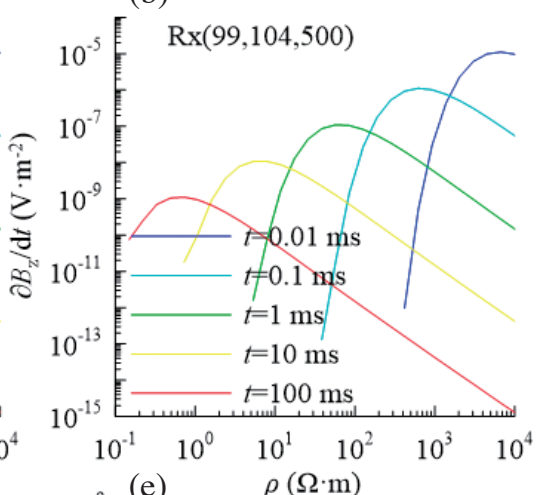

(e) $\quad \rho(\Omega \cdot \mathrm{m})$

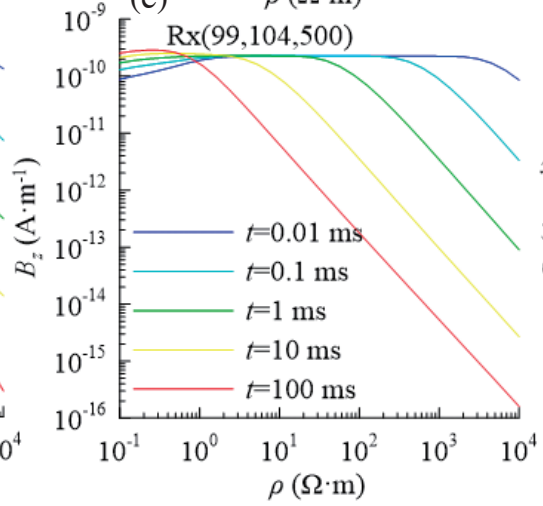

(c)
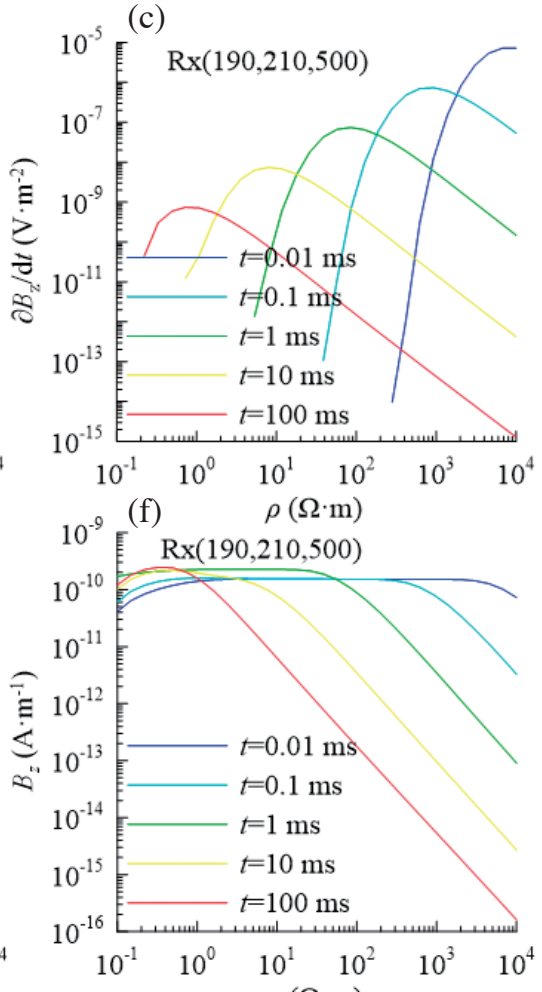

Fig. 4. Response curves of $\partial B_{z} / d t$ and $B_{z}$ receiving point at different offsets; (a) $\operatorname{Rx}(0,0,500), \partial B_{z} / d t$; (b) $\operatorname{Rx}(99,104,500), \partial B_{z} / d t ;$ (c) $\operatorname{Rx}(190$, $210,500), \partial B_{z} / d t ;$ (d) $\operatorname{Rx}(0,0,500), B_{z} ;$ (e) $\operatorname{Rx}(99,104,500), B_{z} ;$ (f) $\operatorname{Rx}(190,210,500), B_{z}$. 


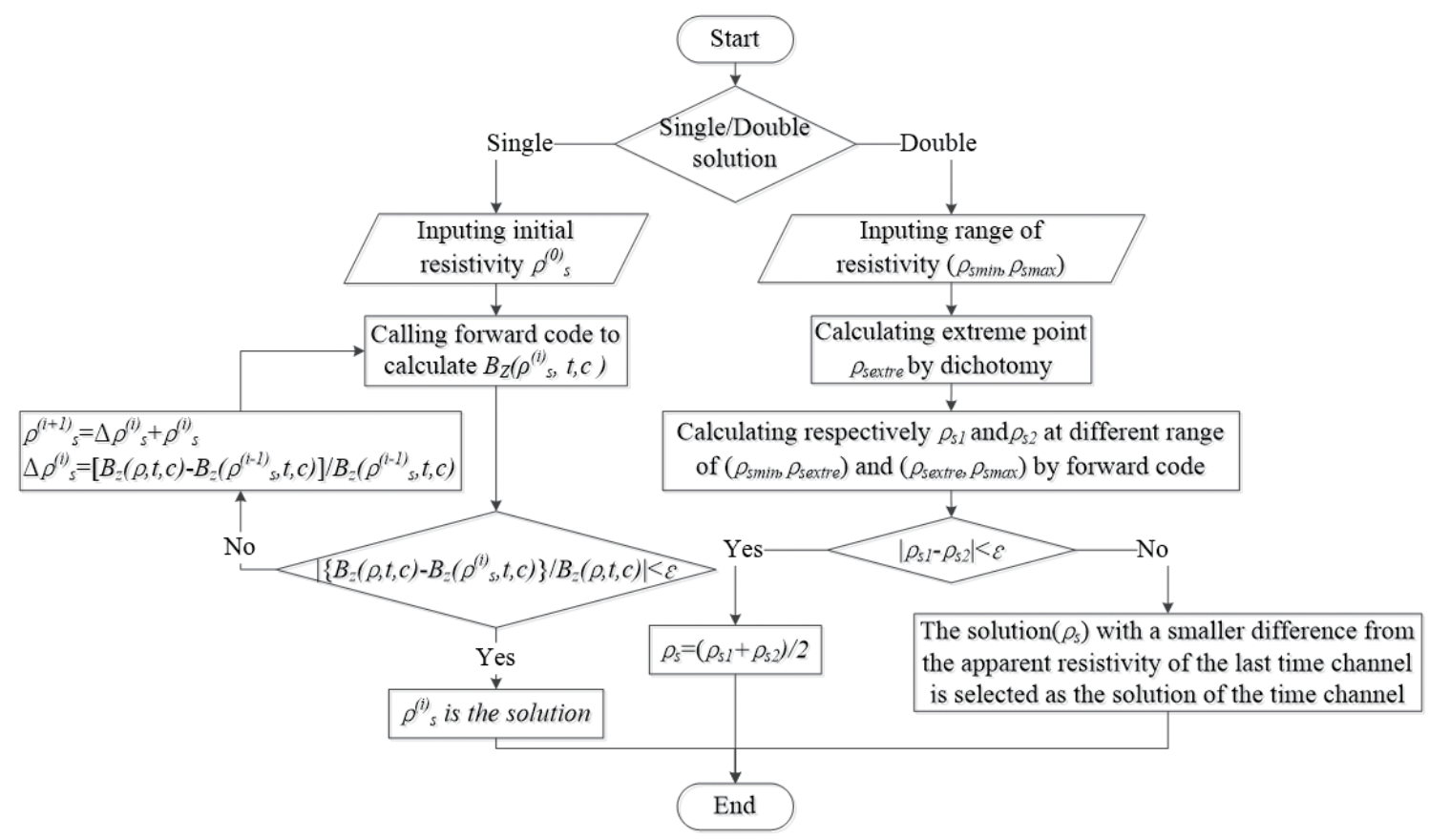

Fig. 5. Flow chart of full-domain apparent resistivity calculation of Ground-Tunnel TEM.
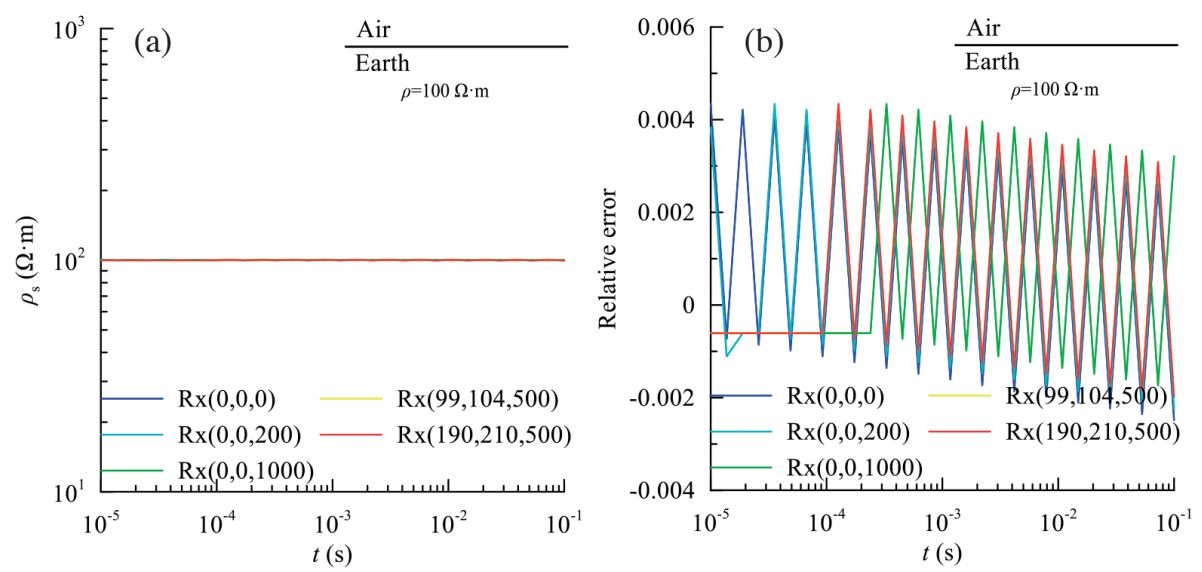

Fig. 6. Full-domain apparent resistivity and relative error curves of homogeneous earth model of Ground-Tunnel TEM; (a) full-domain apparent resistivity; (b) relative error. 
a series of one-dimensional models is selected for calculation and analysis in this paper: $\mathrm{H}, \mathrm{K}, \mathrm{A}$, and Q geoelectric models. This paper further analyses the response characteristics of the full-domain apparent resistivity curves of receiving points changing with the stratum parameters at different depths and offsets according to the method features of electromagnetic wave emission in ground launching and electromagnetic wave receiving in the tunnel.

\subsection{Analysis of Calculation Examples of Full-Domain Apparent Resistivity}

Four geoelectric models have been designed to change second stratum resistivity. Figures 7 to 10 show the fulldomain apparent resistivity curves of ground-tunnel TEM in the $\mathrm{H}, \mathrm{K}, \mathrm{A}$, and $\mathrm{Q}$ geoelectric models. By comparing the curves of the four geoelectric models, we can be that the full-domain apparent resistivity curves of all four geoelectric models can reflect the electric structure of corresponding stratum when the receiving point is located on the earth's surface. The starting and ending branches of the curves approximate the resistivity values of the first and third strata respectively. The curves diverge in the middle. The response amplitude of the full-domain apparent resistivity curve increases with the increase in the differences between the second stratum resistivity values and the first and third stratum resistivity values.

When the receiving point is located within the first stratum, the full-domain resistivity curve shape is roughly consistent with the curve shape when the receiving point is located on the surface. Compared to the receiving point on the earth's surface, this receiving point is close to the second stratum. The full-domain apparent resistivity curve can reflect the electric information of the second stratum earlier, and the response amplitude is greater.

When the receiving point is located within the second stratum, the first branch of the full-domain apparent resistivity curve will change as the second stratum resistivity changes and the change trend of the branch is the same as that of the second stratum resistivity. The full-domain apparent resistivity can reflect the electric information of the second stratum relatively earlier, and its response amplitude is greater.

When the receiving point is located within the third stratum, the first branch of the full-domain apparent resistivity approximates the third stratum resistivity. With the increase of observation time, the apparent resistivity curve quickly shows the electric information of the second stratum, but the response amplitude will reduce. As the observation time increases, a flat section will appear in the apparent resistivity curve. This flat section can be used to judge (a)

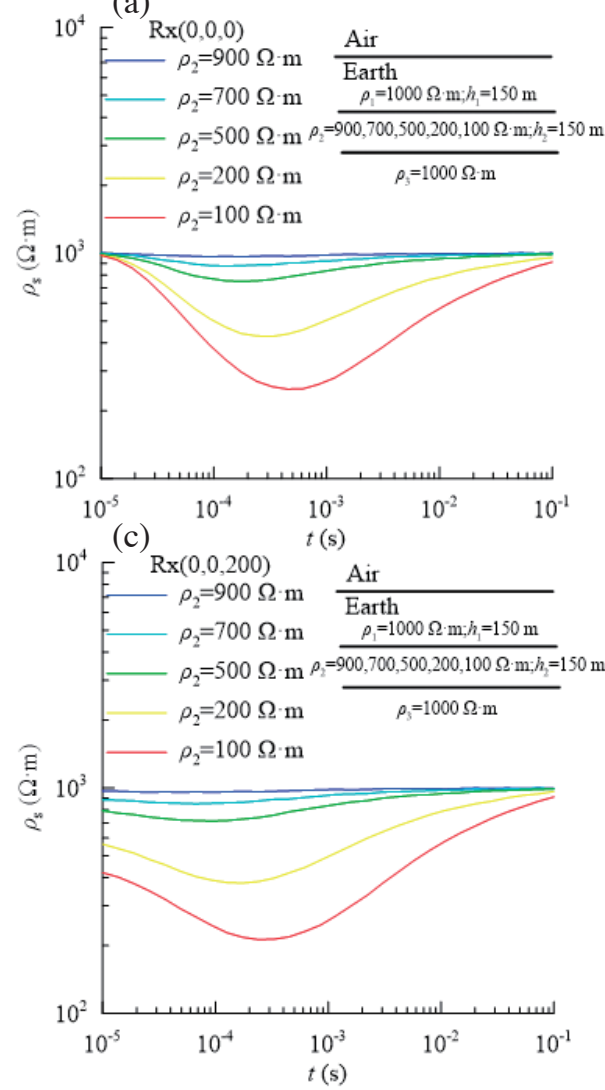

(b)

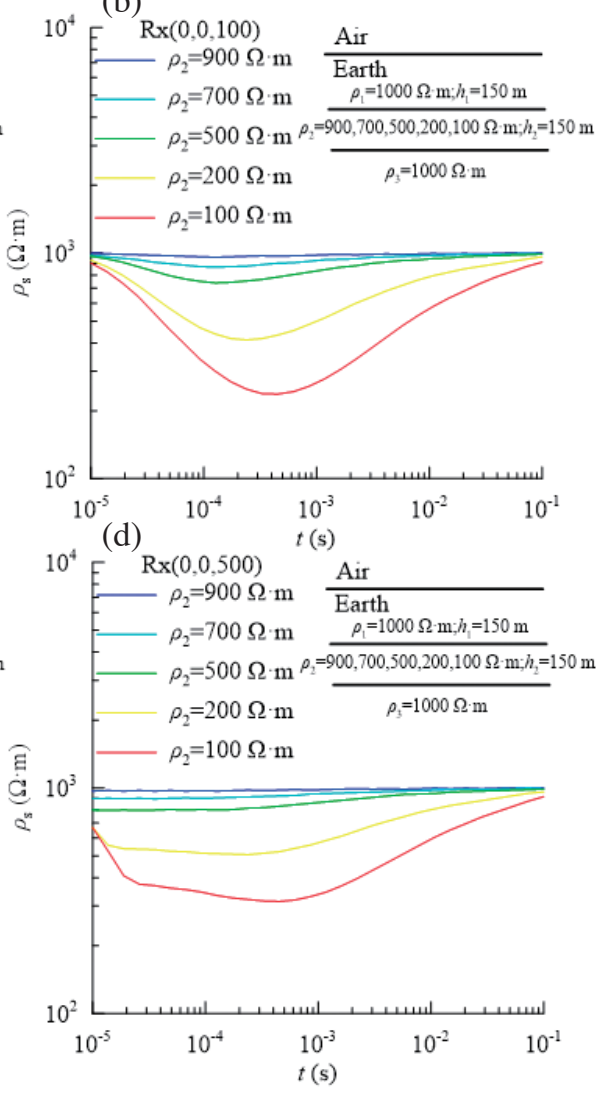

Fig. 7. Full-domain apparent resistivity curves of $\mathrm{H}$ type geoelectric model; (a) $\operatorname{Rx}(0,0,0)$; (b) $\operatorname{Rx}(0,0,100) ;(\mathrm{c}) \operatorname{Rx}(0,0,200)$; (d) $\mathrm{Rx}(0,0,500)$. 
(a)
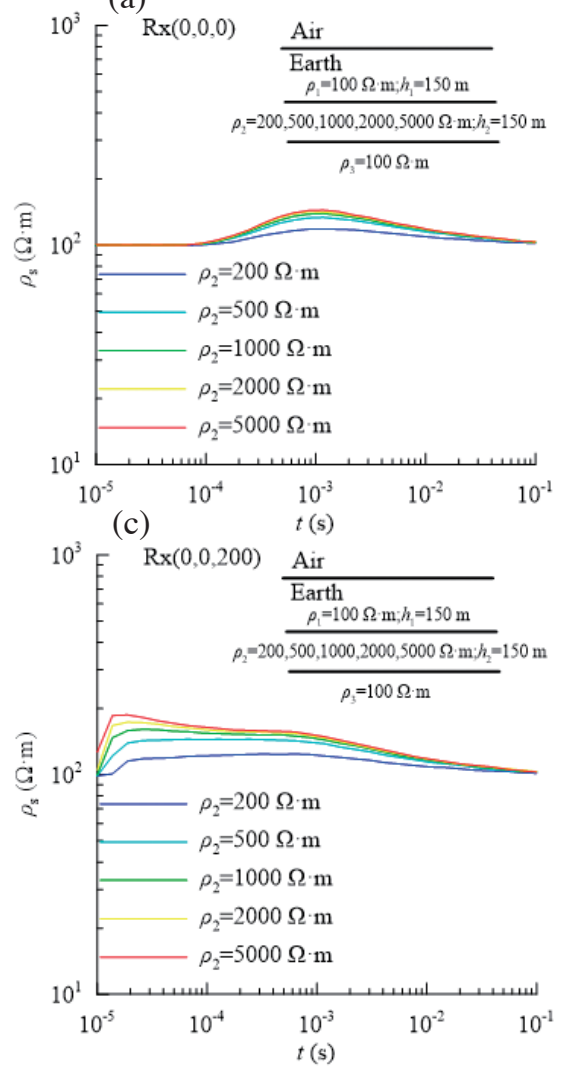

(b)
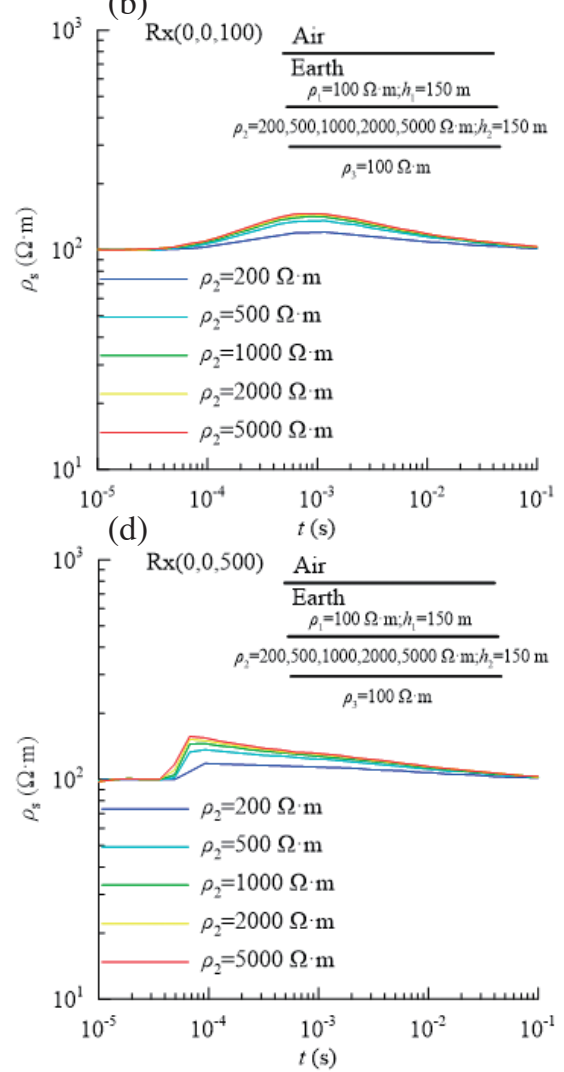

Fig. 8. Full-domain apparent resistivity curves of K type geoelectric model; (a) $\operatorname{Rx}(0,0,0)$; (b) $\operatorname{Rx}(0,0,100)$; (c) $\operatorname{Rx}(0,0,200)$; (d) $\operatorname{Rx}(0,0$, 500).

(a)

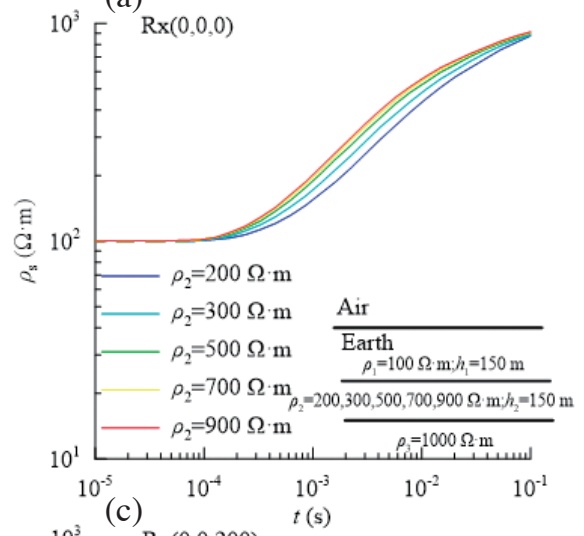

(c)

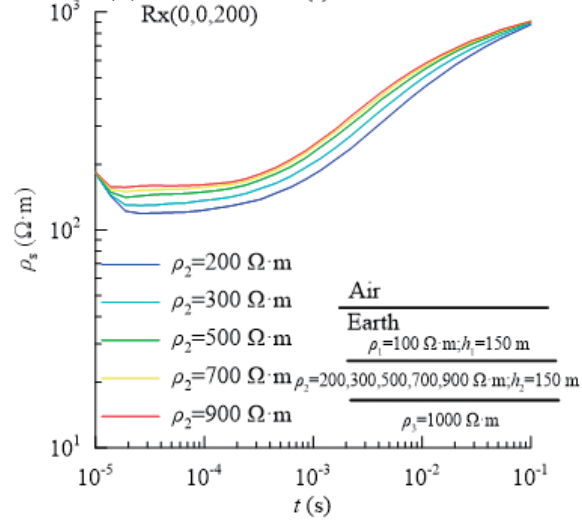

(b)
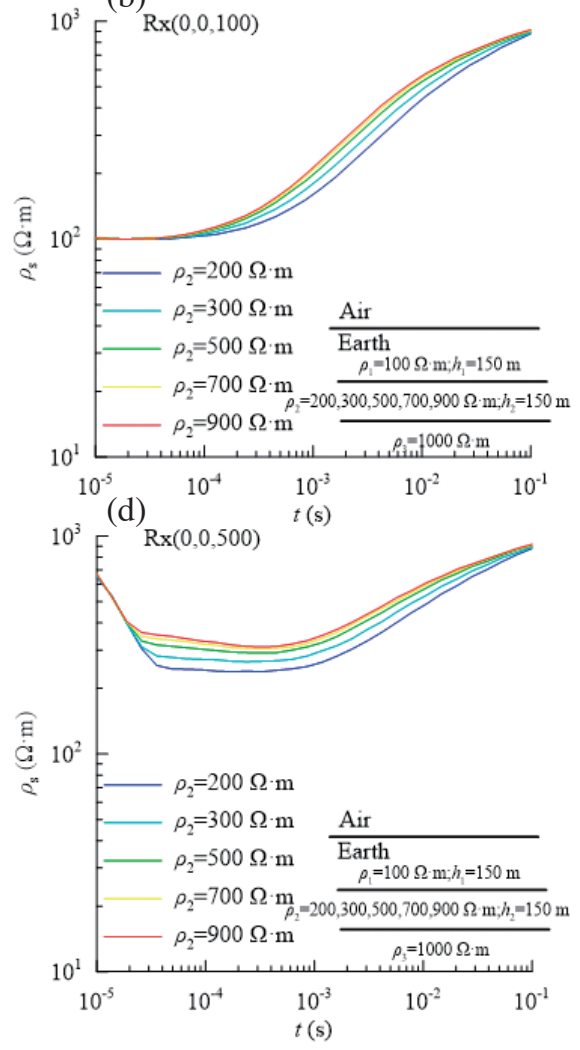

Fig. 9. Full-domain apparent resistivity curves of A type geoelectric model; (a) $\operatorname{Rx}(0,0,0)$; (b) $\operatorname{Rx}(0,0,100) ;(c) \operatorname{Rx}(0,0,200)$; (d) $\operatorname{Rx}(0,0,500)$. 
(a)

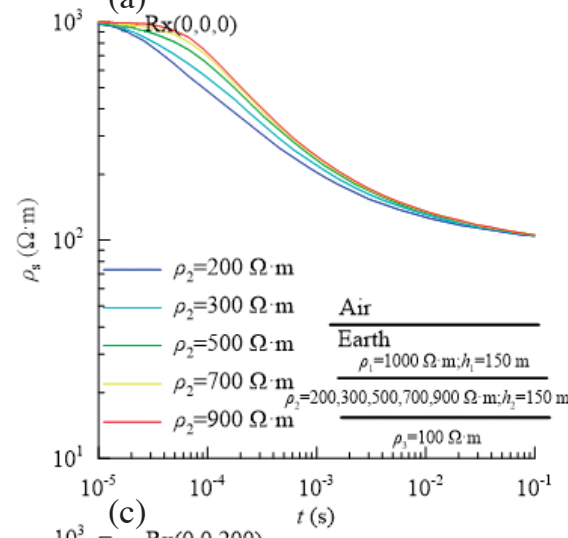

$10^{3}-\operatorname{Rx}(0,0,200)$

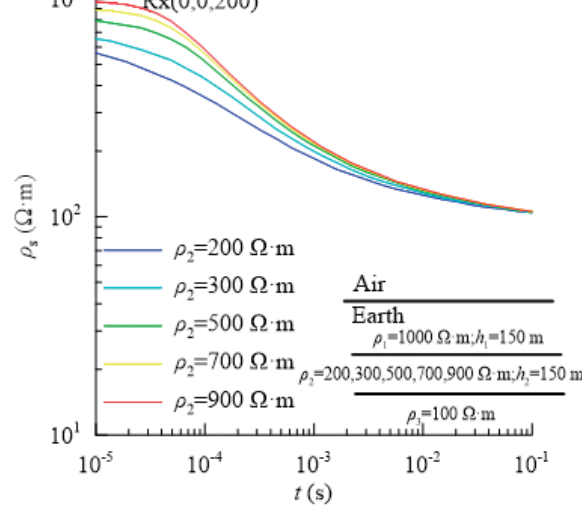

(b)
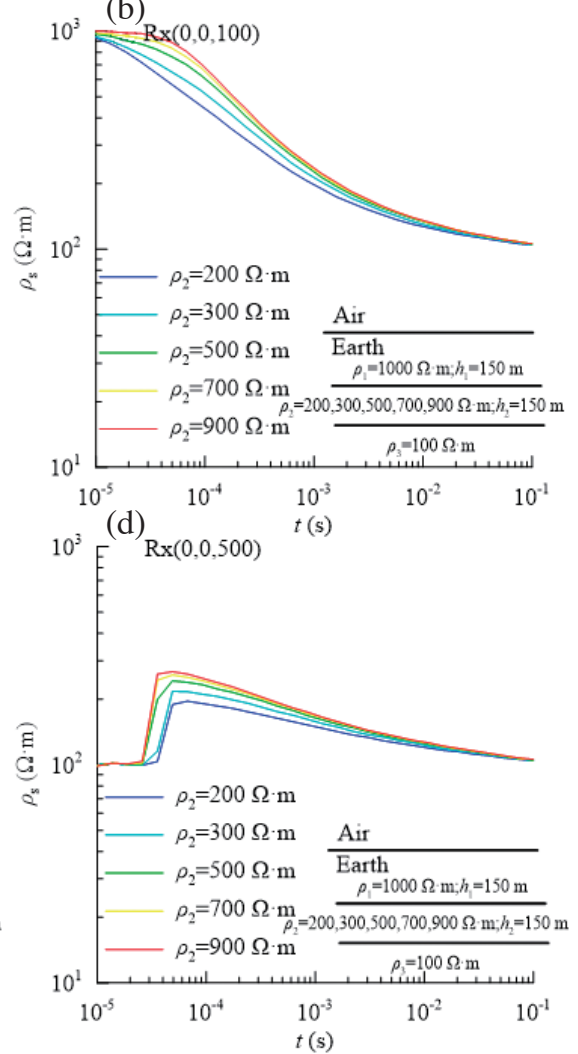

Fig. 10. Full-domain apparent resistivity curves of Q type geoelectric model; (a) $\operatorname{Rx}(0,0,0)$; (b) $\operatorname{Rx}(0,0,100) ;(\mathrm{c}) \operatorname{Rx}(0,0,200)$; (d) $\operatorname{Rx}(0,0,500)$.

the thickness of the second stratum. The ending branch then approximates to the third stratum resistivity value.

It can be observed from the above curve characteristics that the depth at the receiving point greatly affects the fulldomain apparent resistivity curve of this method, but it can reflect the electric structure of the corresponding stratum.

\subsection{Influences of Offset on Full-Domain Apparent Resistivity}

By combining the characteristics of the receiving devices in this method, the receiving point is placed at different strata $z=0,100,200,500 \mathrm{~m}$. The full-domain apparent resistivity is simply computed when the offsets are different. The coordinates of the receiving points are $(0,0, z),(50$, $50, z),(99,104, z)$, and $(190,210, z)$.

This paper has selected the full-domain apparent resistivity curves of the ground-tunnel TEM on the H-type geoelectric cross section and K-type geoelectric cross section with obvious curve forms at different offsets. As shown in Figs. 11 and 12, when the receiving points are located in different strata, the full-domain apparent resistivity curve shapes with different offsets are consistent and the differences among them are minor. This indicates that full-domain apparent resistivity of ground-tunnel TEM calculated using vertical magnetic induction intensity is applicable to the measurement when the offsets are different.

\section{CONCLUSIONS}

(1) Based on the monotonic relationship between vertical magnetic induction intensity and apparent resistivity, an idea for finding a unique solution and/of multiple solutions for the full-domain apparent resistivity of groundtunnel TEM is proposed according to the inverse function theorem, allowing the calculation of the full-domain apparent resistivity of ground-tunnel TEM.

(2) The response characteristics of the full-domain apparent resistivity curves of different geoelectric models changing in accordance with the stratum parameters are analysed when the receiving points are located within different strata. When the stratum parameters and receiving point positions change, all the changes of the full-domain apparent resistivity curves will be reflected in their time sequences and amplitudes. The characteristic of full-domain apparent resistivity curves of this method can effectively reflect stratum information.

(3) Regarding the calculation of full-domain apparent resistivity at different offsets, the full-domain apparent resistivity curve shapes are consistent and the differences 
(a)

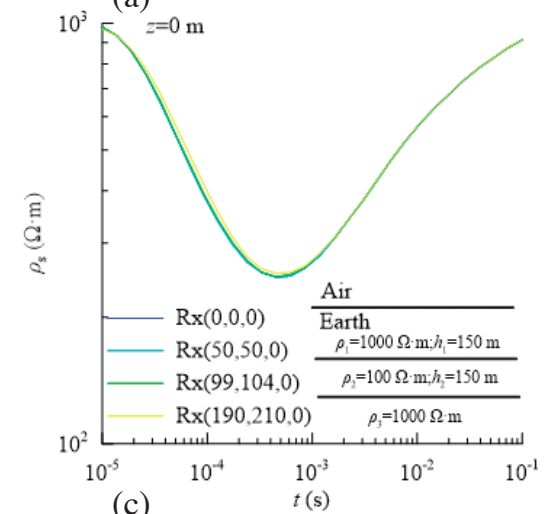

(c)

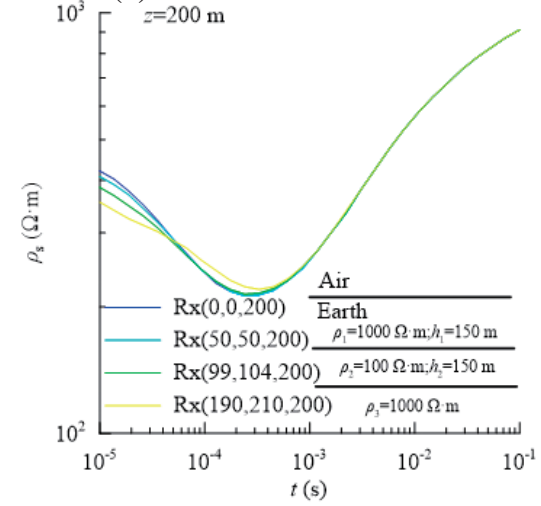

(b)

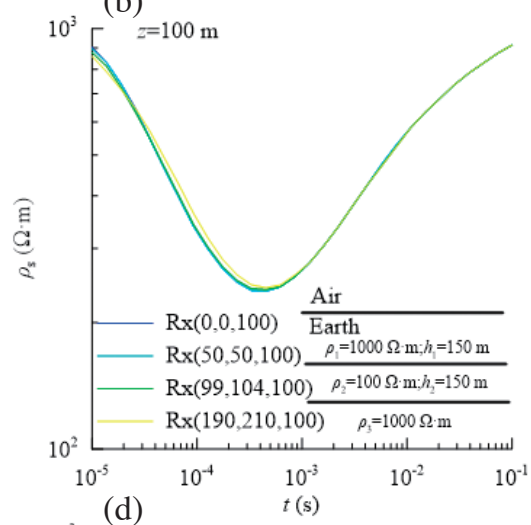

(d)

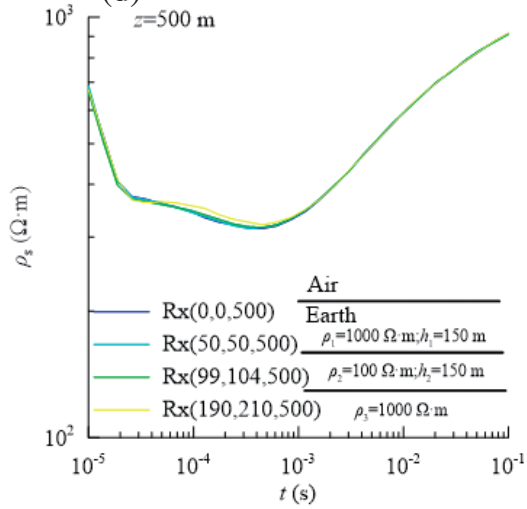

Fig. 11. Full-domain apparent resistivity curves of $\mathrm{H}$ type geoelectric model at different offsets; (a) $z=0 \mathrm{~m}$; (b) $z=100 \mathrm{~m}$; (c) $z=200 \mathrm{~m}$; (d) $z=500 \mathrm{~m}$.

(a)

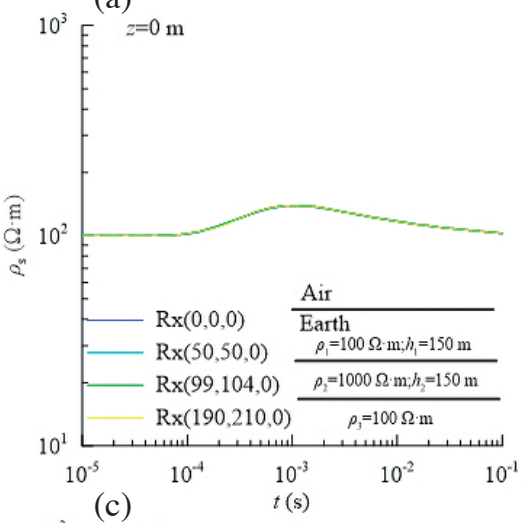

(c)

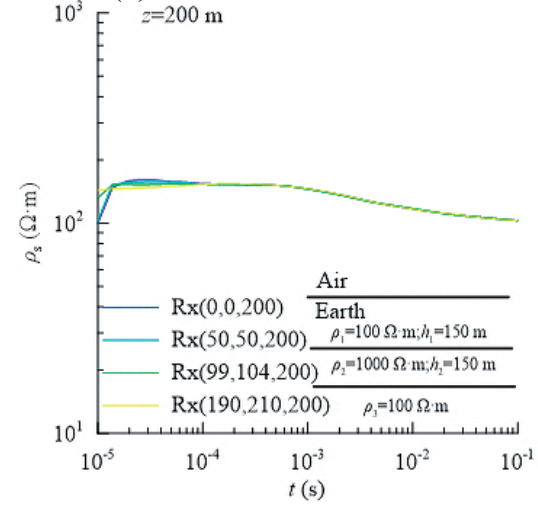

(b)

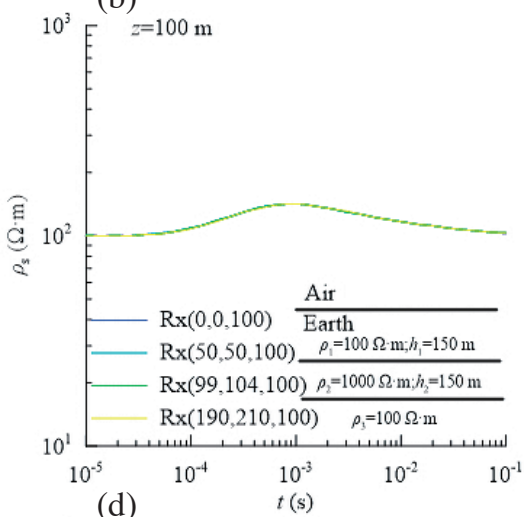

(d)

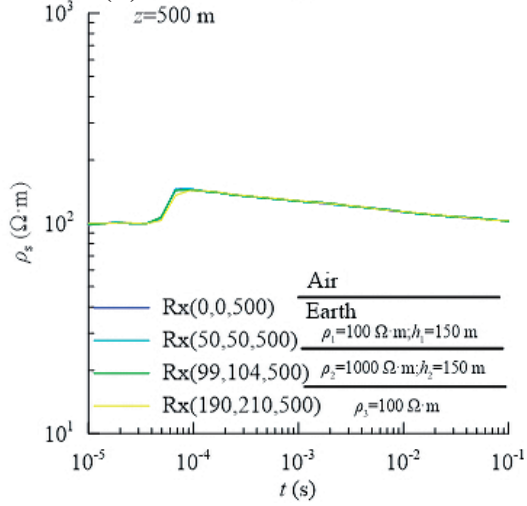

Fig. 12. Full-domain apparent resistivity curves of K type geoelectric model at different offsets; (a) $z=0 \mathrm{~m}$; (b) $z=100 \mathrm{~m}$; (c) $z=200 \mathrm{~m}$; (d) $z=500 \mathrm{~m}$. 
among them are minor, which indicates that this interpretation method is applicable to geological prospecting with different offsets.

(4) Because the receiving points of ground-tunnel TEM are located in underground tunnels, the characteristics of the full-domain apparent resistivity curves are greatly affected by the depths of the receiving points. The characteristic of full-domain apparent resistivity curves at different receiving point depths provides theoretical references for the practical applications of this method.

Acknowledgements This research was supported by the National Key R\&D Program of China under Grant (2018YFC0807802-3). In addition, we are particularly grateful for the advice and help of editors and reviewers.

\section{REFERENCES}

Bai, D., M. A. Meju, J. Lu, L. Wang, and Z. He, 2003: Numerical calculation of all-time apparent resistivity for central loop transient electromagnetic method. Chin.J. Geophys., 46, 998-1010, doi: 10.1002/cjg2.419. [Link]

Chang, J.-H., J.-C. Yu, and Z.-X. Liu, 2016: Three-dimensional numerical modeling of full-space transient electromagnetic responses of water in goaf. Appl. Geophys., 13, 539-552, doi: 10.1007/s11770-016-0572-y. [Link]

Chen, X.-D., Y. Zhao, J. Zhang, G.-Y. Lü, P. Ma, and Y.D. Dai, 2012: The applications of HTc SQUID magnetometer to TEM. Chin. J. Geophys., 55, 702-708, doi: 10.6038/j.issn.0001-5733.2012.02.034. [Link]

Cui, J., G. Xue, W. Chen, and J. Deng, 2015: Calculation of all-time apparent resistivity for B field due to electrical source TEM. Progress in Geophysics, 30, 2690-2697. (in Chinese)

Jiao, X., 2016: Study on the Ground-Tunnel Transient Electromagnetic Method Technology. China University of Mine and Technology, $119 \mathrm{pp}$.

Jiao, X., S. Liu, Z. Jiang, and X. Liu, 2016: Response characteristics of water-conductive strike fault by mine transient electromagnetic advanced detection. Coal Engineering, 48, 92-94. (in Chinese)

Li, F., G. Liao, and X. Liu, 2013: Application of mine transient electromagnetic method in coal mine collapse area detection. Safety in Coal Mines, 4, 155-158, doi: 10.13347/j.cnki.mkaq.2013.04.001. (in Chinese) [Link]

Li, J., Z. Zhu, S. Liu, X. Liu, S. Zeng, and W. Luo, 2011: Rectangular loop transient electromagnetic field expressed by Gaver-Stehfest algorithm. Oil Geophysical Prospecting, 46, 489-492.

Li, J., S. Liu, X. Jiao, Z. Jiang, and X. Hu, 2015: ThreeDimensional Forward Modeling of Ground-Well Transient Electromagnetic Method. Oil Geophysical Prospecting, 50, 556-564, doi: 10.13810/j.cnki. issn.1000-7210.2015.03.025. (in Chinese) [Link]

Li, S., K. Li, M. Zhai, H. Sun, and S. Wang, 2016: Analysis of grounded Transient Electromagnetic with surfacetunnel configuration in mining. Journal of China Coal Society, 41, 2024-2032.

Liu, Y., 2014: Study on the application effect of mine transient electromagnetic technology under the metal interference. Master Thesis, China university of mine and technology, $82 \mathrm{pp}$.

Meng, Q.-X. and H.-P. Pan, 2012: Numerical simulation analysis of surface-hole TEM responses. Chin. J. Geophys., 55, 1046-1053, doi: 10.6038/j.issn.00015733.2012.03.035. (in Chinese) [Link]

Qi, Z., X. Li, W. Guo, Y. Liu, and Q. Wu, 2011a: Definition of apparent resistivity for horizontal-component of transient electromagnetic method. Journal of China Coal Society, 36, 88-93, doi: 10.13225/j.cnki. jccs.2011.s1.002. [Link]

Qi, Z., X. Li, H. Zhu, B. Ma, J. Zhang, and Q. Wu, 2011b: Definition of apparent resistivity for non-center vertical component of Large-loop TEM configuration. Progress in Geophysics, 26, 1350-1358, doi: 10.3969/j. issn.1004-2903.2011.04.028. (in Chinese) [Link]

Qiang, J., Y. Luo, J. Tang, and Y. Li, 2010: The algorithm of all-time apparent resistivity for Airborne Transient Electromagnetic (ATEM) survey. Progress in Geophysics, 25, 1657-1661. (in Chinese)

Wang, T.-P., Y.-T. Chen, C.-C. Chen, T.-H. Tung, S.-N. Cheng, and C.-Y. Yu, 2020: Application of cross-hole electrical resistivity tomography to groundwater contaminated remediation site. Terr. Atmos. Ocean. Sci., 31, 507-521, doi: 10.3319/TAO.2019.06.17.01. [Link]

Wu, Q., 2014: Progress, problems and prospects of prevention and control technology of mine water and reutilization in China. Journal of China Coal Society, 39, 795-805, doi: 10.13225/j.cnki.jccs.2014.0478. [Link]

Xue, G., C. Bai, S. Yan, S. Greenhalgh, M. Li, and N. Zhou, 2012: Deep Sounding TEM investigation method based on a modified fixed central-loop system. J. Appl. Geophys., 76, 23-32, doi: 10.1016/j.jappgeo.2011.10.007. [Link]

Xue, G., W. Chen, and S. Yan, 2018a: Research study on the short offset time-domain electromagnetic method for deep exploration. J. Appl. Geophys., 155, 131-137, doi: 10.1016/j.jappgeo.2018.05.019. [Link]

Xue, G., X. Li, S. Yu, W. Chen, and Y. Ji, 2018b: The application of ground-airborne TEM systems for underground cavity detection in China. Journal of Environmental and Engineering Geophysics, 23, 103-113.

Xue, G., W. Chen, Z. Ma, and D. Hou, 2018c: Identifying deep saturated coal bed zones in China through the use of large loop TEM. Journal of Environmental and Engineering Geophysics, 23, 135-142.

Yen, N.-T., T.-L. Lin, L.-R. Liao, P.-Y. Chang, and H. 
Mittal, 2020: Illustrate mud-fluid conduits and their variety using resistivity image profiling method in Southwest Taiwan. Terr. Atmos. Ocean. Sci., 31, 523537, doi: 10.3319/TAO.2019.07.02.01. [Link]

Yin, C. and H. Piao, 1991: A study of the definition of apparent resistivity in electromagnetic sounding. Geophysical \& Geochemical Exploration, 15, 290-299. (in Chinese)

Zhao, Y., G. Xue, S. Huang, Z. Lu, J. Cui, and D. Hou, 2016: Test and analysis of TEM $B$ field parameter.
Geophysical and Geochemical Exploration, 40, 174178, doi: 10.11720/wtyht.2016.1.31. [Link]

Zhou, X., S. Liu, J. Chang, S. Zhou, Y. Wang, D. Wei, and Y. Liu, 2014: Influences on metal support to mine transient electromagnetic detection and correction technology. Coal Science and Technology, 42, 101-104.

Zhu, N., 2015: Research on 1D forward and inversion interpretation method of transient electromagnetic detection between ground and tunnel. China University of Mine and Technology, 69 pp. 